\title{
ASYMPTOTIC BEHAVIOR OF TRAVELING WAVE SOLUTIONS OF THE EQUATIONS FOR THE FLOW OF A FLUID WITH SMALL VISCOSITY AND CAPILLARITY*
}

BY

\section{J. L. BOLDRINI}

Brown University

Abstract. We study the oscillations of the traveling wave solutions of

$$
\left\{\begin{array}{l}
u_{t}=v_{x}, \\
v_{t}=-p(u)_{x}+\varepsilon v_{x x}-\delta u_{x x x}
\end{array}\right.
$$

for small $\varepsilon$ and $\delta$. These solutions give information about the structure of the shock layers in fluids with small viscosity and capillarity. We conclude that the traveling wave has oscillations with increasing amplitude when $\varepsilon$ and $\delta$ approach zero such that $\delta \neq O\left(\varepsilon^{2}\right)$. When $\delta=o\left(\varepsilon^{2}\right)$, if there are oscillations, their amplitude decreases to zero as $\varepsilon$ and $\delta$ approach zero. When $\delta=\varepsilon^{2}$ the shape of the traveling wave is independent of the magnitude of $\varepsilon$ and $\delta$.

1. Introduction. We study the qualitative behavior of the traveling wave solutions of the equations for the one-dimensional flow of a fluid with small viscosity and capillarity. Several authors (van der Waals [7], Korteweg [4], Slemrod [5], Hagan and Slemrod [2]) have stressed the importance of the capillarity for an understanding of the motion of materials when sharp changes of density occur on a small spatial scale, as in shock and phase transition layers. In this work we consider the simplest model for a fluid with viscosity and capillarity for which the equations of motion, in Lagrangian coordinates, are

$$
\left\{\begin{array}{l}
u_{t}=v_{x} \\
v_{t}=-p(u)_{x}+\varepsilon v_{x x}-\delta u_{x x x},
\end{array}\right.
$$

where $u(x, t)$ is the specific volume and $v(x, t)$ is the velocity at time $t$ of a material point with reference position $x, p(\cdot)$ is the pressure, and $\varepsilon$ and $\delta$ are positive parameters measuring the strength of viscosity and capillarity, respectively.

The behavior of the traveling wave solutions of (1.1), for small $\varepsilon$ and $\delta$, gives the structure that viscosity and capillarity impose on shock layers of shock-wave solutions of

\footnotetext{
* Received May 14, 1985.
} 
the system

$$
\left\{\begin{array}{l}
u_{t}=v_{x}, \\
v_{t}=-p(u)_{x} .
\end{array}\right.
$$

In this work we will be assuming that the pressure function satisfies

$$
p(\cdot) \text { is } C^{2} \text {-smooth, } p(\cdot)>0, p^{\prime}(\cdot)<0, p^{\prime \prime}(\cdot)>0 \text {. }
$$

In particular, these conditions imply that the system (1.2) is strictly hyperbolic.

We will prove the following:

THEOREM 1.1. Corresponding to any shock-wave solution (1.2), there is a traveling wave solution of (1.1) connecting the states to the left and to the right of the shock. When $\delta=\varepsilon^{2}$, the shape of the traveling wave is independent of the magnitudes of $\varepsilon$ and $\delta$; when $\delta \neq o\left(\varepsilon^{2}\right)$, the traveling wave has oscillations and the amplitude of the oscillations grows as $\varepsilon$ and $\delta$ approach zero; when $\delta=o\left(\varepsilon^{2}\right)$, the traveling wave is monotone or, if it has oscillations, the amplitude of the oscillations decreases to zero as $\varepsilon$ and $\delta$ approach zero.

The above result explains the following: the system (1.1) was studied in [1] by a different approach. In that paper, the author proved that there is a subsequence of the family of the solutions $\left\{\left(u_{\varepsilon}^{\delta}, v_{\varepsilon}^{\delta}\right), \varepsilon, \delta \downarrow 0\right\}$ of (1.1), corresponding to any fixed initial condition, that converges almost everywhere to a solution of (1.2), with the same initial condition, provided that $\delta=O\left(\varepsilon^{2}\right)$ and there is $L^{\infty}$-stability of the family of solutions. Roughly speaking, the proof of this result consisted in finding enough estimates to pass from weak-* convergence to almost everywhere convergence. Theorem 1.1 stated above indicates that the condition $\delta=O\left(\varepsilon^{2}\right)$ is probably necessary to obtain the result in [1] and that it is the appearance of oscillations with large amplitude that prevents the conclusion of almost everywhere convergence when $\delta \neq O\left(\varepsilon^{2}\right)$.

The proof of Theorem 1.1 will be split in two parts: in the first one (Sec. 2) we prove the existence of the traveling waves, and in the second (Sec. 3) we study their qualitative behavior.

2. Existence of traveling waves. We start our analysis by recalling that the shock-wave solutions of (1.2) (terminology according to [6]) correspond to the solutions of the Riemann problem for (1.2) with initial conditions $(u(x, 0), v(x, 0))=\left(u^{+}, v^{+}\right)$for $x>0$ and $(u(x, 0), v(x, 0))=\left(u^{-}, v^{-}\right)$for $x<0$, where the state $\left(u^{+}, v^{+}\right)$is required to be on the shock curve through $\left(u^{-}, v^{-}\right)$. This yields as solution a shock wave, connecting the states $\left(u^{-}, v^{-}\right)$and $\left(u^{+}, v^{+}\right)$, with speed of propagation $U$ given by the Rankine-Hugoniot condition asscciated with the system (1.2). According to $U>0$ or $U<0$, the solution is called a front-shock or a back-shock, respectively.

To find the traveling wave solution of (1.1) connecting the states $\left(u^{-}, v^{-}\right)$and $\left(u^{+}, v^{+}\right)$, we let

$$
u(x, t)=\hat{u}(z), \quad v(x, t)=\hat{v}(z)
$$


where $z=(x-U t) / \delta^{k}$. $U$ is the shock speed and $k$ is to be determined through the analysis. The equations that $\hat{u}(z), \hat{v}(z)$ satisfy are

$$
\left\{\begin{array}{l}
-U \frac{d \hat{u}}{d z}=\frac{d \hat{v}}{d z} \\
-U \frac{d \hat{v}}{d z}=-\frac{d}{d z} p(\hat{u})+\varepsilon \delta^{-k} \frac{d^{2} \hat{v}}{d z^{2}}-\delta^{1-2 k} \frac{d^{3} \hat{v}}{d z^{3}} .
\end{array}\right.
$$

Choosing $k=1 / 2$, the above system can be reduced to the single equation

$$
U^{2} \frac{d \hat{u}}{d z}=-\frac{d}{d z} p(\hat{u})-\varepsilon \delta^{-1 / 2} U \frac{d^{2} \hat{u}}{d z^{2}}-\frac{d^{3} \hat{u}}{d z^{3}} .
$$

But we also have the requirement

$$
\left\{\begin{array}{l}
\lim _{z \rightarrow-\infty} \hat{u}(z)=u^{-}, \\
\lim _{z \rightarrow-\infty} \hat{v}(z)=v^{-}, \\
\lim _{z \rightarrow+\infty} \hat{u}(z)=u^{+}, \\
\lim _{z \rightarrow+\infty} \hat{v}(z)=v^{+},
\end{array}\right.
$$

and, integrating (2.1) from $-\infty$ to $z$, we obtain

$$
\frac{d^{2} \hat{u}}{d z^{2}}=f(\hat{u})-\varepsilon \delta^{-1 / 2} U \frac{d \hat{u}}{d z}
$$

where $f(\hat{u})=-p(\hat{u})+p\left(u^{-}\right)-U^{2}\left(\hat{u}-u^{-}\right)$.

From now on, to simplify the notation, we drop the circumflex from $\hat{u}$ and denote it simply by $u$.

Introducing the variable $w=d u / d z$, the previous equation is equivalent to the system

$$
\left\{\begin{array}{l}
\frac{d u}{d z}=w \\
\frac{d w}{d z}=f(u)-\varepsilon \delta^{-1 / 2} U w
\end{array}\right.
$$

where $f(u)=-p(u)+p\left(u^{-}\right)-U^{2}\left(u-u^{-}\right)$.

We observe that the Rankine-Hugoniot conditions and (1.3) imply that $\left(u^{+}, 0\right)$ and $\left(u^{-}, 0\right)$ are the only equilibrium points of this system.

Now we split our analysis in two cases:

First case: front-shocks. According to [6, p. 307] we have $0<\left(-p^{\prime}\left(u^{+}\right)\right)^{1 / 2}<U<$ $\left(-p^{\prime}\left(u^{-}\right)\right)^{1 / 2}$, which implies:

$$
\left\{\begin{array}{l}
U^{2}+p^{\prime}\left(u^{+}\right)>0 \\
U^{2}+p^{\prime}\left(u^{-}\right)<0
\end{array}\right.
$$

and, since $p^{\prime \prime}(\cdot)>0$,

$$
u^{-}<u^{+}
$$

To analyze the critical point $\left(u^{-}, 0\right)$, we linearize $(2.3)$ around it:

$$
\frac{d}{d z}\left[\begin{array}{l}
x \\
y
\end{array}\right]=\left[\begin{array}{ll}
0 & 1 \\
-p\left(u^{-}\right)-U^{2} & -\varepsilon \delta^{-1 / 2} U
\end{array}\right]\left[\begin{array}{l}
x \\
y
\end{array}\right] .
$$


The characteristic polynomial is $\lambda^{2}+\varepsilon \delta^{-1 / 2} U \lambda+\left(p^{\prime}\left(u^{-}\right)+U^{2}\right)=0$ and the eigenvalues are

$$
\lambda_{ \pm}=\frac{-\varepsilon \delta^{-1 / 2} U}{2}+\left[\frac{\left(\varepsilon \delta^{-1 / 2} U\right)^{2}}{4}+\left(-p^{\prime}\left(u^{-}\right)-U^{2}\right)\right]^{1 / 2},
$$

with corresponding eigenvectors $\left(1, \lambda_{ \pm}\right)$. Since $\lambda_{+}$and $\lambda_{-}$have opposite signs [by (2.4)] we conclude that the equilibrium point $\left(u^{-}, 0\right)$ is a saddle point with unstable manifold tangent to $\left(1, \lambda_{+}\right)$. Moreover, the facts that for any $a, b>0$,

$$
-\frac{a}{2}+\left(\frac{a^{2}}{4}+b^{2}\right)^{1 / 2}<b,
$$

$\varepsilon \delta^{-1 / 2} U>0$ (front-shock) and $-p\left(u^{-}\right)-U^{2}>0$ (by 2.4 ) imply that

$$
\lambda_{+}<\left(-p^{\prime}\left(u^{-}\right)-U^{2}\right)^{1 / 2} .
$$

Now, it can be easily verified that the function

$$
E(u, w)=\frac{w^{2}}{2}+\int_{u^{+}}^{u}\left[p(s)-p\left(u^{-}\right)+U^{2}\left(s-u^{-}\right)\right] d s
$$

is a Liapunov function for (2.3) such that, when $(u(z), w(z))$ is a solution,

$$
\frac{d}{d z} E(u(z), w(z))=-\varepsilon \delta^{-1 / 2} U w^{2} \leqslant 0 .
$$

Also, the assumptions (1.3) imply that there is a unique $l>u^{+}$such that

$$
\int_{u^{-}}^{l}\left[p(s)-p\left(u^{-}\right)+U^{2}\left(s-u^{-}\right)\right] d s=0 .
$$

Eq. (2.8) can be visualized as the requirement that the areas of the shaded regions I and II in Fig. 2.1 are the same.

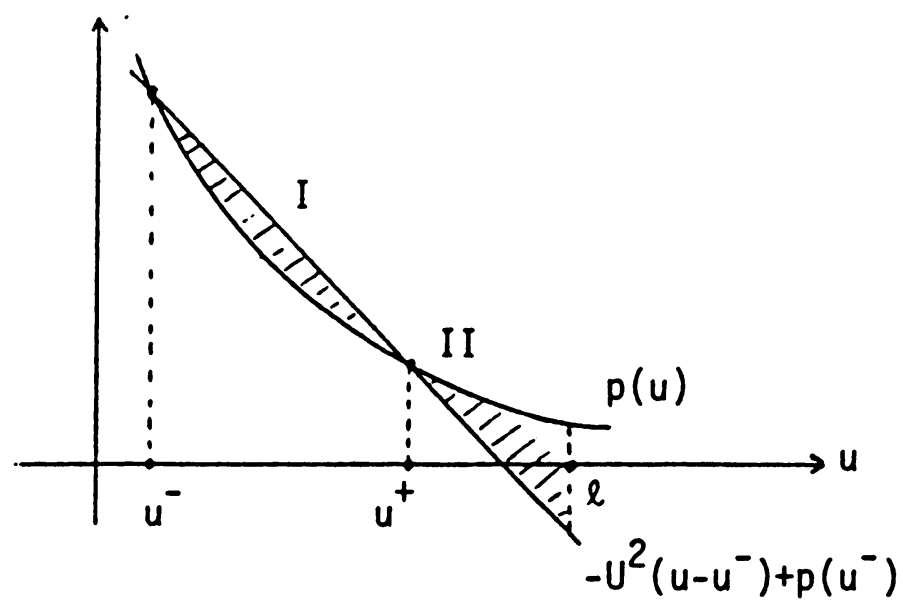

FIG. 2.1 
Now we define the following set in the phase plane:

$$
\begin{aligned}
\Omega & =\left\{(u, w): u_{-} \leqslant u \leqslant l, E(u, w) \leqslant E\left(u^{-}, 0\right)\right\}, \\
& =\left\{(u, w): u_{-} \leqslant u \leqslant l, w^{2} \leqslant 2 \int_{u}^{u^{-}}\left[p(s)-p\left(u^{-}\right)+U^{2}\left(s-u^{-}\right)\right] d s\right\},
\end{aligned}
$$

whose boundaries are given by the following curves:

$$
\begin{array}{r}
\Gamma_{ \pm}=\left\{(u, w): w=w_{ \pm}(u)= \pm \sqrt{2}\left[\int_{u}^{u^{-}}\left[p(s)-p\left(u^{-}\right)+U^{2}\left(s-u^{-}\right)\right] d s\right]^{1 / 2},\right. \\
\left.u^{-} \leqslant u \leqslant l\right\} .
\end{array}
$$

For later use, we observe that the slope of $\Gamma_{+}$at $\left(u^{-}, 0\right)$ is

$$
\frac{d w_{+}}{d u}\left(u^{-}\right)=\left(-p^{\prime}\left(u^{-}\right)-U^{2}\right)^{1 / 2}>0 \quad[\text { by }(2.4)],
$$

and the slope of $\Gamma_{-}$at $\left(u^{-}, 0\right)$ is

$$
\frac{d w_{-}}{d u}\left(u^{-}\right)=-\left(-p^{\prime}\left(u^{-}\right)-U^{2}\right)^{1 / 2}<0 \quad[\text { by (2.4)] }
$$

Moreover, (2.8) implies that $w_{+}(l)=w_{-}(l)=0$ and $\Omega$ can be pictured in the phase plane as in Fig. 2.2.

Now, (2.6) and (2.9) imply that the orbit $\gamma$ of (2.3) leaving $\left(u^{-}, 0\right)$, along the unstable manifold, enters the interior of $\Omega$, and (2.7) implies that it cannot leave it. Using the LaSalle invariance principle [3] and the fact that we have only two equilibrium points, we conclude that the $\omega$-limit set of $\gamma$ has to be an equilibrium point. Since $\gamma$ cannot approach $\left(u^{-}, 0\right)$ because this would imply that $E(u, w)$ had to increase [which is prevented by (2.7)], we conclude that the $\omega$-limit set of $\gamma$ is $\left(u^{+}, 0\right)$; that is, $\gamma$ connects $\left(u^{-}, 0\right)$ and $\left(u^{+}, 0\right)$. Therefore, we have the required traveling wave connecting the states $\left(u^{-}, v^{-}\right)$and $\left(u^{+}, v^{+}\right)$.

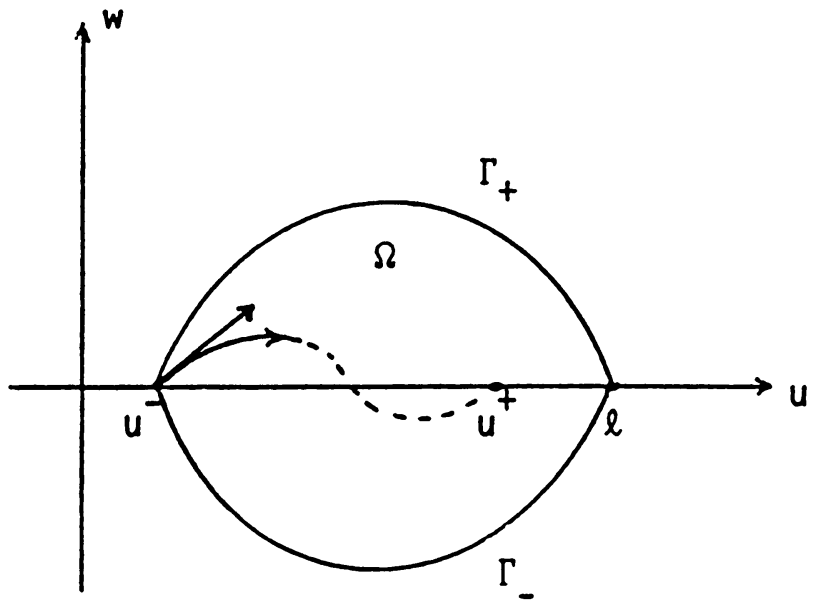

FIG. 2.2 
We observe that the existence of such traveling wave is independent of the ratio between $\varepsilon$ and $\delta$.

Second case: back-shocks. The argument is similar to the one used in the previous case. According to [6, p. 307] we must have $-\left(-p^{\prime}\left(u^{+}\right)\right)^{1 / 2}<U<-\left(-p^{\prime}\left(u^{-}\right)\right)^{1 / 2}<0$, which implies

$$
\left\{\begin{array}{l}
p^{\prime}\left(u^{+}\right)+U^{2}<0 \\
p^{\prime}\left(u^{-}\right)+U^{2}>0
\end{array}\right.
$$

and since $p^{\prime \prime}(\cdot)>0$,

$$
u^{+}<u^{-} .
$$

Linearization around the equilibrium point $\left(u^{+}, 0\right)$, yields the system:

$$
\frac{d}{d t}\left[\begin{array}{l}
x \\
y
\end{array}\right]=\left[\begin{array}{ll}
0 & 1 \\
-p^{\prime}\left(u^{+}\right)-U^{2} & -\varepsilon \delta^{-1 / 2} U
\end{array}\right]\left[\begin{array}{l}
x \\
y
\end{array}\right] .
$$

The characteristic polynomial is $\lambda^{2}+\varepsilon \delta^{-1 / 2} U \lambda+\left(\left(p^{\prime} u^{+}\right)+U^{2}\right)=0$ and the eigenvalues are

$$
\tilde{\lambda}_{ \pm}=-\frac{\left(\varepsilon \delta^{-1 / 2} U\right)}{4} \pm\left[\frac{\left(\varepsilon \delta^{1 / 2} U\right)^{2}}{4}+\left(-p^{\prime}\left(u^{+}\right)-U^{2}\right)\right]^{1 / 2},
$$

with corresponding eigenvectors $\left(1, \tilde{\lambda}_{ \pm}\right)$.

As in the first case, (2.4) implies that $\left(u^{+}, 0\right)$ is a saddle point.

The facts that for any $a<0, b>0$,

$$
-\frac{a}{2}-\left(\frac{a^{2}}{4}+b^{2}\right)^{1 / 2}>-b,
$$

$\varepsilon \delta^{-1 / 2} U<U$ (back-shock), and $-p^{\prime}\left(u^{+}\right)-U^{2}>0$ imply that

$$
0>\tilde{\lambda}_{-}>-\left(-p^{\prime}\left(u^{+}\right)-U^{2}\right)^{1 / 2} \text {. }
$$

Now we consider the function

$$
F(u, w)=\frac{w^{2}}{2}+\int_{u^{-}}^{u}\left[p(s)-p\left(u^{-}\right)-U^{2}\left(s-u^{-}\right)\right] d s
$$

and observe that along an orbit of (2.3)

$$
\frac{d F}{d z}=-\varepsilon \delta^{-1 / 2} U w^{2} \geqslant 0
$$

As in the first case, we observe that the assumptions (1.3) imply that there is a unique $i>u^{-}$such that

$$
\int_{u^{+}}^{i}\left[p(s)-p\left(u^{-}\right)+U^{2}\left(s-u^{-}\right)\right] d s=0
$$

(with a similar geometric interpretation).

Now we define the following set in the phase plane:

$$
\begin{aligned}
\tilde{\Omega} & =\left\{(u, w): u^{+} \leqslant u \leqslant i ; F(u, w) \leqslant F\left(u^{+}, 0\right)\right\} \\
& =\left\{(u, w): u^{+} \leqslant u \leqslant i ; w^{2} \leqslant 2 \int_{u}^{u^{+}}\left[p(s)-p\left(u^{-}\right)+U^{2}\left(s-u^{-}\right)\right] d s\right\},
\end{aligned}
$$


whose boundaries are given by the curves

$$
\tilde{\Gamma}_{ \pm}=\left\{(u, w): u^{+} \leqslant u \leqslant \tilde{l}, \tilde{w}_{ \pm}(u),\right.
$$

where

$$
\left.\tilde{w}_{ \pm}(u)= \pm \sqrt{2}\left[\int_{u}^{u^{+}}\left[p(s)-p\left(u^{-}\right)+U^{2}\left(s-u^{-}\right)\right] d s\right]^{1 / 2}\right\} .
$$

For later use, we observe that the slope of $\tilde{\Gamma}_{+}$at $\left(u^{+}, 0\right)$ is

$$
\frac{d \tilde{w}_{+}}{d u}\left(u^{+}\right)=\left(-p^{\prime}\left(u^{+}\right)-U^{2}\right)^{1 / 2}>0 \quad[\text { by }(2.11)],
$$

and the slope of $\tilde{\Gamma}_{-}$at $\left(u^{+}, 0\right)$ is

$$
\frac{d \tilde{w}_{-}}{d u}\left(u^{+}\right)=-\left(-p^{\prime}\left(u^{+}\right)-U^{2}\right)^{1 / 2}<0 \quad[\text { by }(2.10)] .
$$

By (2.14), we have $\tilde{w}_{+}(\bar{l})=\tilde{w}_{-}(\bar{l})=0$, and so we can picture $\tilde{\Omega}$ in the phase plane as in Fig. 2.3.

Now we observe that (2.15) and (2.12) imply that the orbit $\tilde{\gamma}$ of $(2.3)$ entering in $\left(u^{+}, 0\right)$ along the stable manifold comes from the interior of $\tilde{\Omega}$. (2.3) and the definition of $\tilde{\Omega}$ imply that when we follow $\tilde{\gamma}$ backward in "time" $z$, we cannot leave $\tilde{\Omega}$. Using the LaSalle invariance principle and the fact that we have only two equilibrium points, we conclude that the $\alpha$-limit set of $\tilde{\gamma}$ is a critical point. Since (2.13) implies that such an $\alpha$-limit set cannot be $\left(u^{+}, 0\right)$, we conclude that it is $\left(u^{-}, 0\right)$, and therefore we have a trajectory going from $\left(u^{-}, 0\right)$ to $\left(u^{+}, 0\right)$. This gives a traveling wave connecting the states $\left(u^{-}, v^{-}\right)$and $\left(u^{+}, v^{+}\right)$.

3. Qualitative behavior of the traveling wave. In this section we investigate how the possible oscillations of the traveling wave solutions of (2.3) behave as the parameters $\varepsilon$ and $\delta$ approach zero. Since the front-shock and back-shock cases are similar, we analyze the first one only.

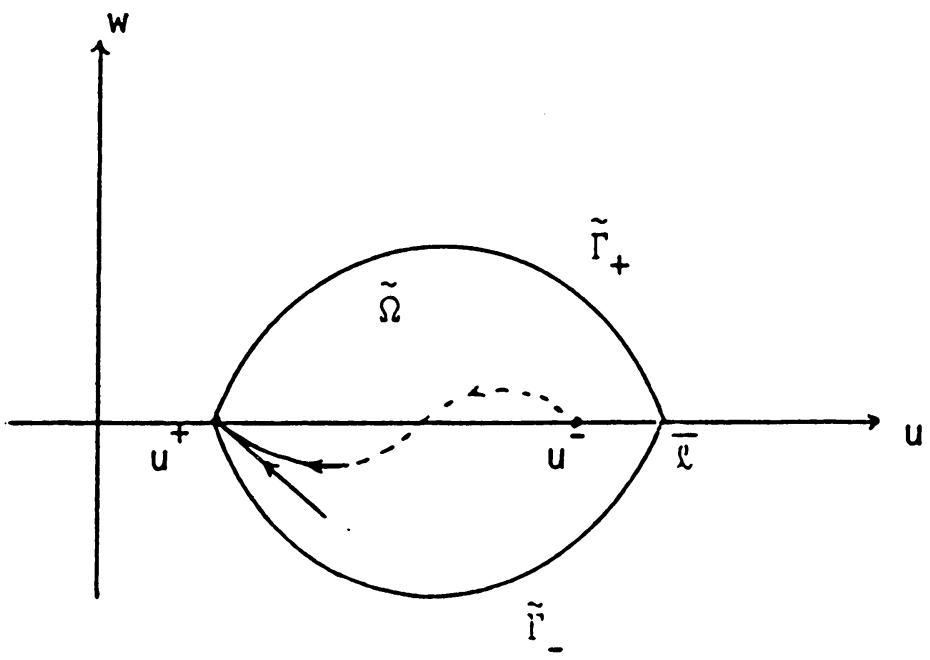

FIG. 2.3 
Consider the situation where a front-shock solution of (1.2) corresponds to the states $\left(u^{-}, v^{-}\right)$and $\left(u^{+}, v^{+}\right)$. Suppose first that $\delta=\varepsilon^{2}$. In this case the system (2.3) is independent of the sizes of $\varepsilon$ and $\delta$, and we conclude that the traveling wave remains invariant as $\varepsilon$ and $\delta$ vary.

In the case where $\delta \neq O\left(\varepsilon^{2}\right)$, we introduce the parameter

$$
\lambda=\varepsilon \delta^{-1 / 2}
$$

and observe that $\lambda \downarrow 0$ as $\varepsilon, \delta \downarrow 0$. Using $\lambda,(2.3)$ is

$$
\left\{\begin{array}{l}
\frac{d u}{d z}=w, \\
\frac{d w}{d z}=f(u)-\lambda U w,
\end{array}\right.
$$

where $f(u)=-p(u)+p\left(u^{-}\right)-U^{2}\left(u-u^{-}\right)$and we recall that $U>0$ because we are in the case of a front-shock.

The system (3.1) is a regular perturbation of the conservative system

$$
\left\{\begin{array}{l}
\frac{d u}{d z}=w, \\
\frac{d w}{d z}=f(u),
\end{array}\right.
$$

for which the orbits are level curves of the function $E(u, w)$ given in Sec. 2. Therefore, the orbit $\gamma_{0}$ that leaves the critical point $\left(u^{-}, 0\right)$ is the level curve given by $E(u, w)=E\left(u^{-}, 0\right)$, and consequently this orbit is transverse to the straight line given by $u=u^{+}$(see Fig. 3.1). By continuity, for small enough $\lambda>0$, the orbit $\gamma(\lambda)$ of (3.1) that leaves the critical point $\left(u^{-}, 0\right)$ along the unstable manifold and enters $\Omega$ (that is, exactly the traveling wave) will cross the line $u=u^{+}$transversely (see Fig. 3.1). Since the flow given by (3.1) points to the right in the upper semiplane, the orbit will have to go around the critical point $\left(u^{+}, 0\right)$ to make the connection.

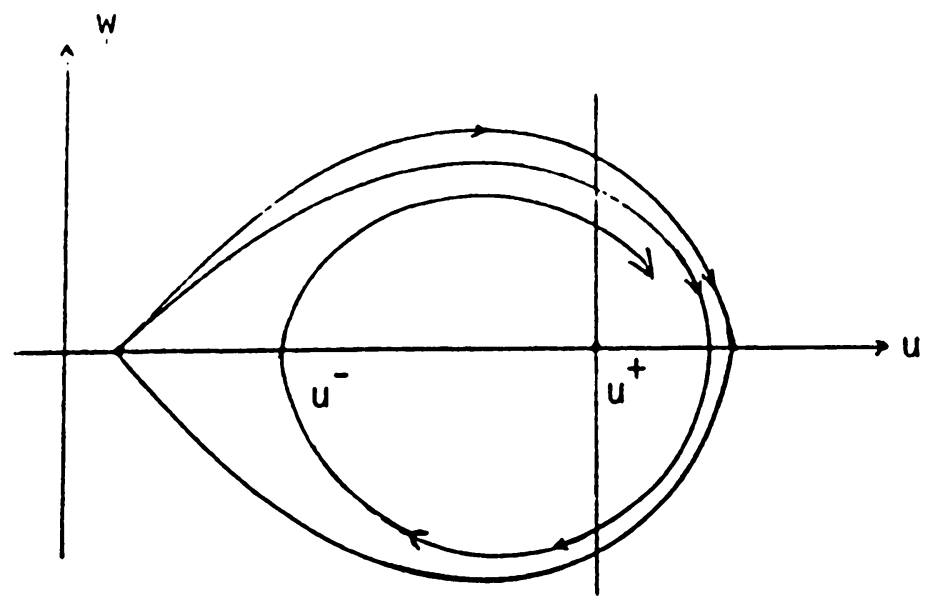

FIG. 3.1 
We conclude that the function $u(z)$ has oscillations around the value $u^{+}$(see Fig. 3.2). Moreover, since $U>0$, we have that

$$
f(u)-\lambda_{1} U w<f(u)-\lambda_{2} U w \quad \text { for } w>0
$$

and

$$
f(u)-\lambda_{1} U w>f(u)-\lambda_{1} U w \quad \text { for } w<0
$$

when $\lambda_{1}>\lambda_{2}>0$. This implies that the traveling waves $\gamma\left(\lambda_{1}\right)$ and $\gamma\left(\lambda_{2}\right)$, corresponding to $\lambda=\lambda_{1}$ and $\lambda=\lambda_{2}$, respectively, are as in Fig. 3.3. Thus, the amplitudes of the oscillations increase as $\lambda \downarrow 0$.

Now let us analyze the case $\delta=o\left(\varepsilon^{2}\right)$. For this we introduce the parameter

$$
\tilde{\lambda}=\varepsilon^{-1} \delta^{1 / 2}
$$

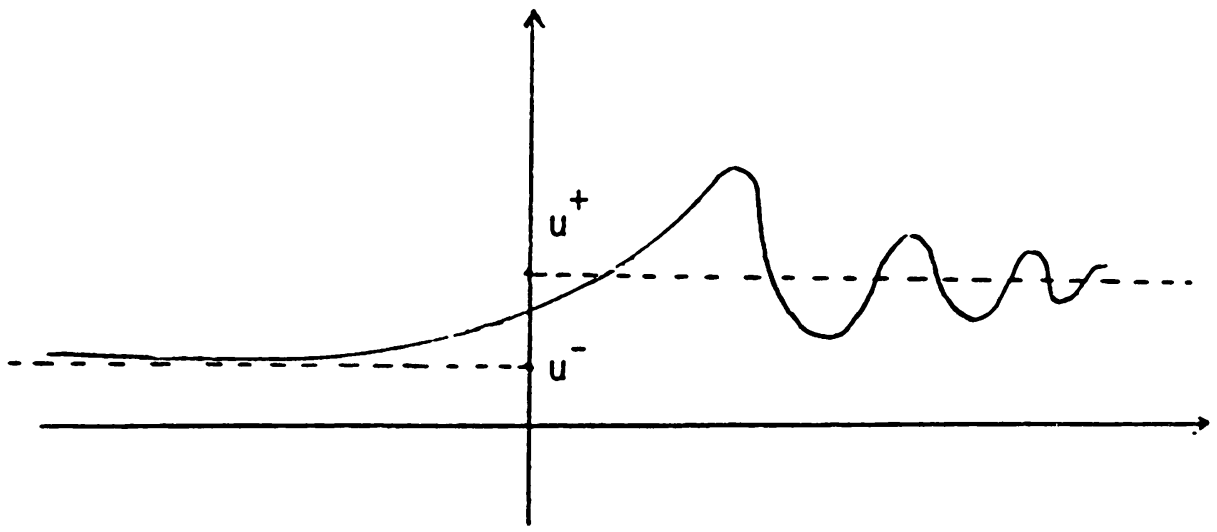

FIG. 3.2

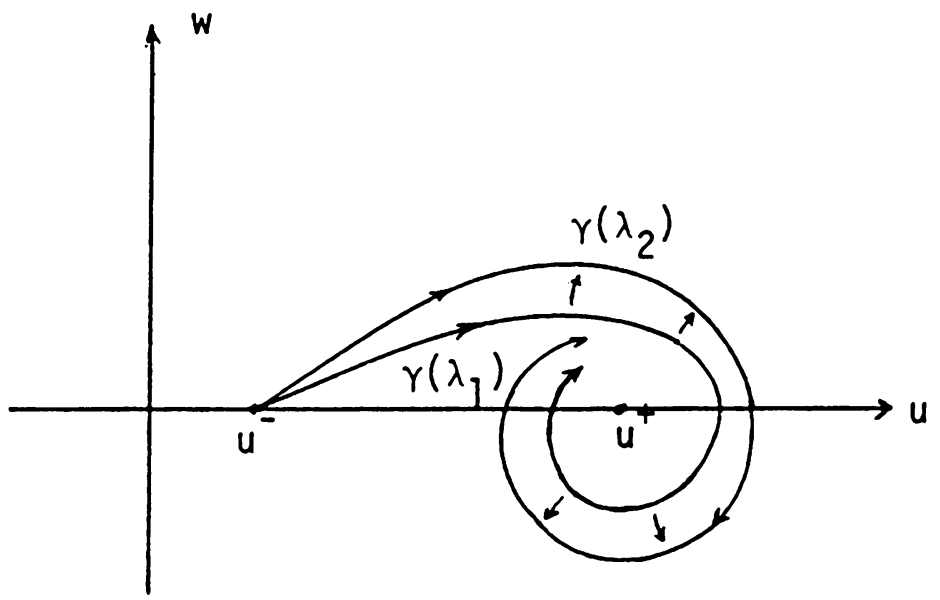

FIG. 3.3 
and observe that $\tilde{\lambda} \downarrow 0$ as $\varepsilon, \delta \downarrow 0$. In terms of $\tilde{\lambda}$, the system (2.3) is

$$
\left\{\begin{array}{l}
\frac{d u}{d z}=w \\
\frac{d w}{d z}=f(u)-\frac{1}{\lambda} U w
\end{array}\right.
$$

where $f(u)$ is the same as in (3.1) and $U>0$.

Now we observe that when $\tilde{\lambda}_{1}>\tilde{\lambda}_{2}>0$, we have

$$
f(u)-\frac{1}{\tilde{\lambda}_{1}} U w>f(u)-\frac{1}{\tilde{\lambda}_{2}} U w \text { for } w>0
$$

and

$$
f(u)-\frac{1}{\tilde{\lambda}_{1}} U w<f(u)-\frac{1}{\tilde{\lambda}_{2}} U w \text { for } w<0 .
$$

This implies that if oscillations are present then the traveling waves $\gamma\left(\tilde{\lambda}_{2}\right)$ and $\gamma\left(\tilde{\lambda}_{1}\right)$, corresponding to $\tilde{\lambda}=\tilde{\lambda}_{1}$ and $\tilde{\lambda}=\tilde{\lambda}_{2}$, respectively, are situated as in Fig. 3.4. Thus, the amplitude of the oscillations (if they exist) decreases as $\tilde{\lambda} \downarrow 0$. Furthermore, we can prove that the amplitude of these oscillations (if they exist) decreases to zero as $\tilde{\lambda} \downarrow 0$. In order to do this, we observe that the vertical component of the field given by (3.2) is negative when $w>\tilde{\lambda} f(u) / U$ and positive otherwise. This implies that

$\left\{\begin{array}{l}\text { the part of the orbit that corresponds to the traveling wave } \\ \text { contained in the semiplane } w \geqslant 0 \text { has points whose ordinates } \\ \text { are no larger than } \tilde{\lambda} M \text { where }\end{array}\right.$

$$
M=\max _{u^{-} \leqslant u \leqslant u^{+}}\{f(u) / U\}
$$

[recall that $f(u)>0$ for $u^{-}<u<u^{+}$].

Since (2.4)(i) implies that $f^{\prime}\left(u^{+}\right)<0$ for a fixed and small enough $r$, we can find constants $k_{2}>k_{1}>0$ such that, for $u \in\left[u^{+}-r, u^{+}+r\right]$,

$$
k_{1}\left[\left(u-u^{+}\right)^{2}+w^{2}\right] \leqslant E(u, w) \leqslant k_{2}\left[\left(u-u^{+}\right)^{2}+w^{2}\right],
$$

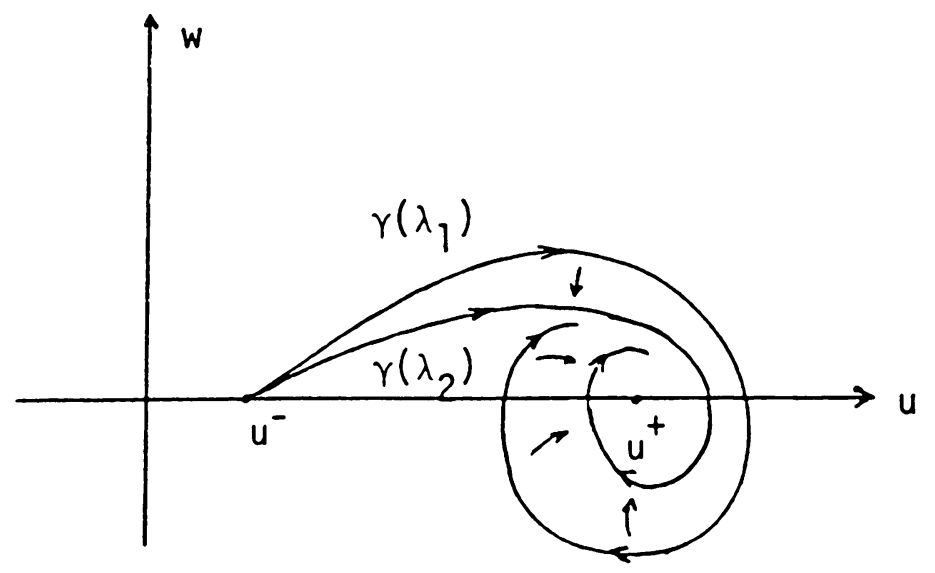

FIG. 3.4 


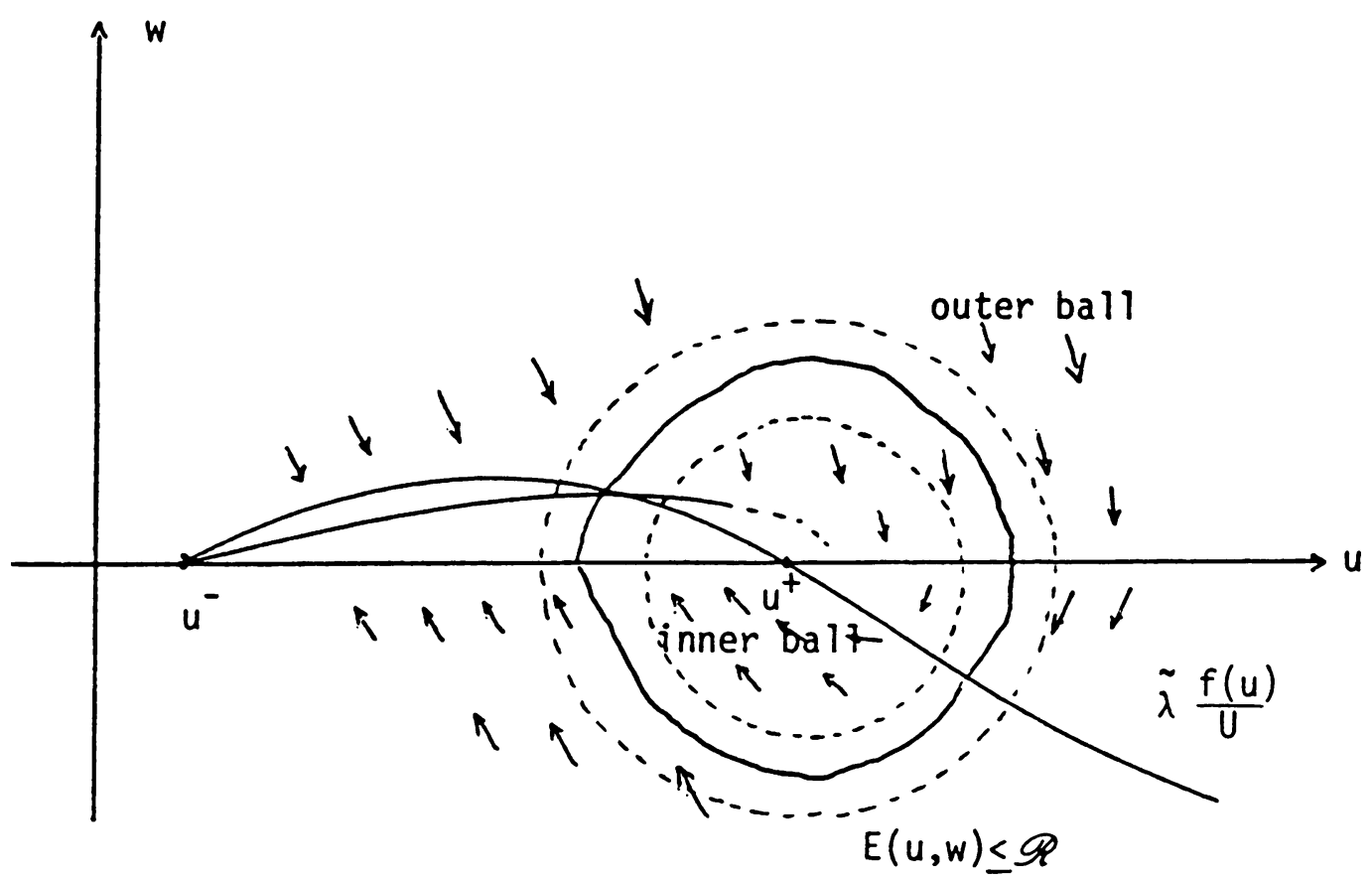

FIG. 3.5

where

$$
E(u, w)=\frac{w^{2}}{2}+\int_{u^{+}}^{u}-f(s) d s
$$

The above inequalities imply that, for $\mathscr{R}$ sufficiently small, the set $\Omega(\mathscr{R})$ defined by

$$
\Omega(\mathscr{R})=\left\{(u, w) \in R^{2}: E(u, w) \leqslant \mathscr{R}\right\}
$$

is contained in the ball with center at $\left(u^{+}, 0\right)$ and radius $\mathscr{R} / k_{1}$ and contains the ball with center at $\left(u^{+}, 0\right)$ and radius $\mathscr{R} / k_{2}$. In the same way as verified for the set $\Omega$ in the previous section, once an orbit enters $\Omega(\mathscr{R})$, it cannot leave it.

If we choose $\mathscr{R}=2 \tilde{\lambda} M k_{1}$, for $\tilde{\lambda}$ sufficiently small, the inner ball in Fig. 3.5 has radius $2 \tilde{\lambda} M$. Since we know that the orbit that corresponds to the traveling wave leaves the critical point $\left(u^{-}, 0\right)$ pointing to the upper semiplane, that while it stays in this semiplane the orbit has to go to the right, and furthermore that it cannot leave this semiplane by crossing the $u$-axis between $u^{-}$and $u^{+}$, from (3.3), we conclude that the orbit that corresponds to the traveling wave enters the inner ball. Therefore, it enters $\Omega\left(2 \tilde{\lambda} M k_{1}\right)$ and cannot leave it.

Since $\Omega\left(2 \tilde{\lambda} M k_{1}\right)$ is positively invariant and is contained in the outer ball, we conclude that the points on this orbit cannot be farther from the critical point $\left(u^{+}, 0\right)$ than the radius of the outer ball, that is, $2 \tilde{\lambda} M k_{1} / k_{2}$, after the orbit enters $\Omega\left(2 \tilde{\lambda} M k_{1}\right)$. 


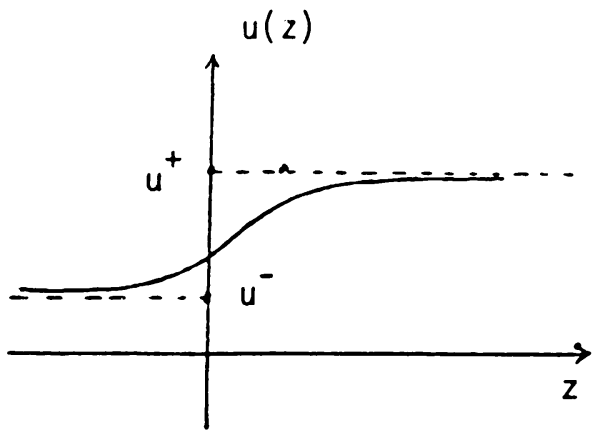

no oscillations

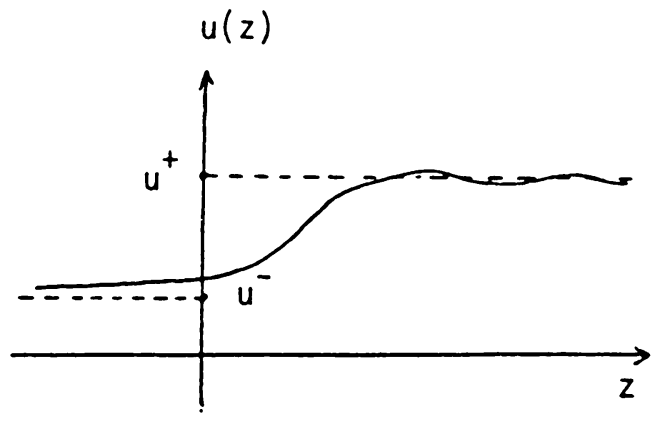

small oscillations

FIG. 3.6

The conclusion is that, if oscillations are present, they satisfy:

$$
\text { amplitude of the oscillations } \leqslant \tilde{\lambda}\left(\frac{2 M k_{1}}{k_{2}}\right) \text {, }
$$

and therefore their amplitude goes to zero as $\varepsilon, \delta \downarrow 0$.

For small $\varepsilon$ and $\delta$, we represent graphically in Fig. 3.6 the two possibilities for the function $u(z)$.

The previous results taken together exactly constitute Theorem 1.1.

Acknowledgment. The author wishes to express his gratitude to Professor C. M. Dafermos for giving several suggestions during the elaboration of this paper.

\section{REFERENCES}

[1] J. L. Boldrini, Is elasticity the proper asymptotic theory for materials with viscosity and capillarity?, Proceedings of the Royal Society of Edinburgh.

[2] R. Hagan and M. Slemrod, The viscosity-capillarity criterion for shocks and phase transitions. Arch. Rat. Mech. Anal. 83, 333-361 (1983)

[3] J. K. Hale, Ordinary differential equations, Krieger, New York (1980)

[4] D. J. Korteweg, Sur la forme que prenent les équations du mouvement des fluids si l'on tient compte des forces capillaires par des variations de densité, Arch. Neerl. Sci. Exactes Nat., Ser. II 6, 1-24 (1901)

[5] M. Slemrod, Admissibility criteria for propagating phase boundaries in a van der Waals fluid, Arch. Rat. Mech. Anal. 81, Number 4, 301-315 (1983)

[6] J. A. Smoller, Shock waves and reaction-diffusion equations, Springer-Verlag, New York (1982)

[7] J. D. van der Waals, Théorie thermodynamique de la capillarité dans l'hypothèse d'une variation continue de densité, Arch. Neerl. Sci. Exactes Nat. 28, 121-209 (1895) 